\title{
Analysis of the Influence of Extended Breastfeeding Care on Maternal Physiological and Psychological Status
}

\author{
Yiyang Xie*, Linan Jia and Yijie Wang \\ Nurse in Beijing Ditan Hospital, Capital Medical University, China
}

*Corresponding author: Yiyang Xie, Nurse in Beijing Ditan Hospital Affiliated to Capital Medical University Hospital Address: No.8, Jingshun East Street, Chaoyang District, Beijing, China

To Cite This Article: Yiyang Xie, Linan Jia, Yijie Wang. Analysis of the Influence of Extended Breastfeeding Care on Maternal Physiological and Psychological Status. Am J Biomed Sci \& Res. 2021 - 13(2). AJBSR.MS.ID.001844. DOI: 10.34297/AJBSR.2021.13.001844.

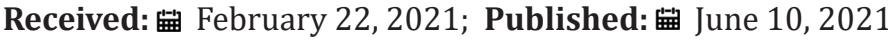

\begin{abstract}
Objective: To analyze the effect of the application of extended breastfeeding care on maternal psychological and physiological status.

Design: A total of 100 parturient women who delivered in our hospital from June 2019 to June 2020 were selected and randomly divided into a control group and a study group, with 50 cases in each group.

Methods: The control group was given routine obstetric care, and the study group was given extended breastfeeding care. The Self-rating Anxiety Scale (SAS) anxiety score, Self-rating Depression Scale (SDS) depression score, success rate of exclusive breastfeeding and incidence of postpartum adverse events were compared between groups.

Results: The SAS anxiety score and SDS depression score of the two groups before nursing were included in the comparison between the two groups, and there was no significant difference ( $\mathrm{P}>0.05)$; after nursing, the study group had significantly lower scores than the control group $(\mathrm{P}<0.05)$. The success rate of pure breastfeeding in the study group was $94 \%$, which was higher than the rate of $72 \%$ in the control group; the incidence of postpartum adverse events in the study group was $6 \%$, which was significantly lower than the rate of $26 \%$ identified in the control group $(\mathrm{P}<0.05)$. Conclusions: The application of extended breastfeeding care can improve the negative emotions of postpartum women, reduce the incidence of postpartum adverse events and improve the success rate of exclusive breastfeeding.
\end{abstract}

Keywords: Extended breastfeeding care; Parturient women; Physiological status; Psychological status

\section{Introduction}

Breastmilk, which contains a large number of trace elements and antibodies, is important for newborns to obtain nutrition and can improve the resistance of newborns to external pathogenic factors; breastmilk is also helpful for growth and development. However, work pressure and the fast pace of life cause many women to return to work soon after delivery, leaving no time to take care of the newborn. As a result, the rate of exclusive breastfeeding is decreasing, and the physical condition of newborns is generally satisfactory [1,2]. Although routine obstetric care can ensure a good breastfeeding rate during hospitalization, after discharge, women receive little attention. We provided extended breastfeeding care for pregnant women to ensure that the pregnant women can be supervised and managed after discharge; thus, the breastfeeding rate was significantly improved. One hundred pregnant women who delivered in our hospital from June 2019 to June 2020 were enrolled to analyze the effect of extended breastfeeding care on maternal psychological and physiological status.

\section{Materials and Methods}

\section{Patient information}

One hundred women who delivered in our hospital from June 2019 to June 2020 were selected; the random number table method was used as a grouping method, and the women were divided into a control group and a study group, with 50 women in 
each group. The characteristics of the women in the study group were as follows: 24-38 years old, with an average age of $31.96 \pm$ 4.82 years; the gestational age was $38-42$ weeks, with an average of $40.34 \pm 1.29$ weeks; 21 multipara and 29 primipara women; 35 natural deliveries; and 15 cesarean sections. The characteristics of the women in the control group were as follows: 25-37 years old, with an average age of $31.93 \pm 4.84$ years; the gestational age was 39-42 weeks, with an average oof $40.36 \pm 1.27$ weeks; 22 multipara and 28 primipara women; 36 natural deliveries; and 14 cesarean sections. There was no significant difference in average age, gestational weeks, history of labor or mode of delivery between the two groups $(\mathrm{P}>0.05)$.

The inclusion criteria were as follows $[2,3]$ :

1. No breastfeeding contraindications, such as AIDS, acute hepatitis B, and active tuberculosis, etc.

2. Single term pregnancy

3. No psychosis, no mental retardation, and able to communicate normally.

4. The pregnant woman and their family were informed of the content of the study, informed consent was signed, and this study met the requirements of medical ethics.

The exclusion criteria were as follows:

1. Premature delivery

2. Breast disease

3. Postpartum depression

4. Malignant tumor, liver and kidney dysfunction and other serious diseases

5. AIDS, acute hepatitis B, active tuberculosis, etc.

\section{Methods}

The control group was given routine obstetric care: Health education on breastfeeding was given to pregnant women after delivery, the women were guided to master the correct nursing posture, a reasonable diet was arranged for pregnant women to ensure adequate nutritional support for postpartum women, the perineum or abdominal incision was recommended to be cleaned frequently to prevent infection, and the requirements of maternal work and rest in order to promote faster recovery were discussed. In the study group, extended breastfeeding care was added. After discharge, nursing staff with strong communication skills and rich nursing experience provided follow-up. The frequency of telephone follow-up was 1 time/week, and the frequency of drop-in follow-up was 1 time/month.
The follow-up included the following $[4,5]$ :

1. The women were guided to have full rest and a reasonable diet; understand wound healing, lochia discharge and pelvic floor muscle recovery; actively communicate with medical staff; understand the problems associated with breastfeeding and receive answers; and receive guidance for correction of wrong behaviors.

2. The objective was to understand the psychological status of the women, to give them encouragement and comfort, to encourage them to breastfeed for at least half a year and guide them to add supplementary food in a timely manner [3].

3. Individualized exercise and diet programs for women were developed to ensure the normal secretion of breast milk and the involution of the uterus as soon as possible.

Maternal family members were guided to give more care and spiritual support to ensure the stability of maternal mood and prevent postpartum depression and postpartum anxiety [5].

Women were followed up with the help of WeChat, QQ and other communication platforms to provide breastfeeding knowledge to the women through the use of messages to improve the breastfeeding skills of the women; the women were able to communicate with the medical staff in the WeChat group or QQ group in a timely manner if they had any questions, and the medical staff could give timely answers.

\section{Main outcome measures}

The Self-rating Anxiety Scale (SAS) anxiety score, Self-rating Depression Scale (SDS) depression score, success rate of exclusive breastfeeding and incidence of postpartum adverse events were compared between groups. The SAS and SDS were used to evaluate anxiety and depression. There were 20 items for both scales; the scores were graded from 1 to 4 , the sum was calculated, rounded, and multiplied by 1.25 to obtain the final score. The higher the score, the more serious the anxiety and depression; if the SAS score is greater than 50 points or the SDS score is greater than 53 points, the individual can be diagnosed with an anxiety disorder or depression, respectively. Postpartum adverse events mainly include constipation, mastitis, uterine involution and so on.

\section{Statistical methods}

All the data were processed by SPSS 22.0 software, the percentage $\mathrm{n}(\%)$ is used to express count data, which were analyzed using the chi-square test; continuous data are presented as the mean \pm standard deviation (SD) ( $\mathrm{x} \pm$ s) and were analyzed by the t-test.

$P<0.05$ indicated significant differences. 


\section{Results}

\section{SAS anxiety score and SDS depression score}

The SAS anxiety score and SDS depression score of the two groups before nursing were included in the comparison between the two groups, and no significant difference was observed $(\mathrm{P}>0.05)$; the two groups were compared again after nursing, and there was a significant difference $(\mathrm{P}<0.05)$ (Table 1$)$.

Table 1: Comparison of SAS Anxiety Score and SDS depression score before and after nursing (points, $x- \pm s$ ).

\begin{tabular}{|c|c|c|c|c|}
\hline \multirow{2}{*}{ Grouping } & \multicolumn{2}{|c|}{ SAS Anxiety Score } & \multicolumn{2}{c|}{ SDS Depression Score } \\
\cline { 2 - 5 } & Before Care & After Care & Before Care & After Care \\
\hline Research group $(\mathrm{n}=50)$ & $55.22 \pm 4.24^{\mathrm{a}}$ & $39.34 \pm 3.13^{\mathrm{a}}$ & $59.21 \pm 6.32^{\mathrm{b}}$ & $42.41 \pm 2.09^{\mathrm{b}}$ \\
\hline Control group $(\mathrm{n}=50)$ & $55.28 \pm 4.31^{\mathrm{c}}$ & $46.18 \pm 4.02^{\mathrm{c}}$ & $59.28 \pm 6.43^{\mathrm{d}}$ & $50.44 \pm 4.23^{\mathrm{d}}$ \\
\hline $\mathrm{t}$ & 0.07 & 9.493 & 0.055 & 12.035 \\
\hline $\mathrm{p}$ & 0.944 & 0 & 0.956 & 0 \\
\hline
\end{tabular}

a.t=21.307, $p=0.000<0.05 ; b .17 .846, p=0.000<0.05 ; c . t=10.918, p=0.000<0.05 ; d . t=8.122, p=0.000<0.05$.

Success rate of exclusive breastfeeding and incidence of postpartum adverse events

The success rate of exclusive breastfeeding in the study group was $94 \%$ higher than that in the control group; the incidence of postpartum adverse events in the study group was $6 \%$, which was lower than the rate of $26 \%$ observed in the control group, and there were significant differences $(\mathrm{P}<0.05)$ (Table 2$)$.

Table 2: Comparison of the success rate of exclusive breastfeeding and the incidence of postpartum adverse events (n, \%).

\begin{tabular}{|c|c|c|c|c|c|}
\hline Grouping & $\begin{array}{c}\text { Success rate of } \\
\text { exclusive breast } \\
\text { feeding }\end{array}$ & Mastitis & Constipation & $\begin{array}{c}\text { Poor uterine } \\
\text { involution }\end{array}$ & Total incidence \\
\hline research group $(\mathrm{n}=50)$ & $47(94.00)$ & $2(4.00)$ & $1(2.00)$ & $0(0.00)$ & $3(6.00)$ \\
\hline Control group $(\mathrm{n}=50)$ & $36(72.00)$ & $5(10.00)$ & $5(10.00)$ & $3(6.00)$ & $13(26.00)$ \\
\hline $\mathrm{x} 2$ & 8.576 & - & - & - & 7.441 \\
\hline $\mathrm{p}$ & 0.003 & - & - & - & 0.006 \\
\hline
\end{tabular}

\section{Discussion}

Antibodies such as IgA in breast milk can improve the immunity of newborns, so breastfeeding is important for newborns. For the postpartum women, the sucking of the newborn can stimulate the maternal body and promote the secretion of prolactin and uterine contraction to accelerate milk secretion, uterine involution and lochia discharge [4,6,7]. However, an increasing number of women are unable to breastfeed for various reasons, which makes postpartum women prone to lochia, uterine involution and infectious diseases. The control group chose to receive routine care at the hospital and did not continue to receive supervision after discharge, so the maternal breastfeeding rate was reduced, and the postpartum women were more prone to adverse emotions and adverse events. The study group received extended breastfeeding care that provided maternal guidance regarding rest, diet, exercise and other aspects to allow them to better recover after delivery. Additionally, regular on-site and telephone follow-up were carried out for pregnant women to urge them to breastfeed. Moreover, realtime contact and health education were carried out via WeChat to improve the physiological status of pregnant women to the greatest extent. In addition, extended breastfeeding care also intervened in the negative psychological state of the pregnant women, such as anxiety and depression. Through health education and psychological nursing, the negative emotions of pregnant women were improved, which can have a positive impact on the recovery of postpartum physiological function and the cooperation degree of the postpartum women so they can recover faster and better after delivery. Moreover, this model also refers to the experience of foreign mothers in extending breastfeeding time and further improve infants and young children's growth and development [8-10]. Our results also suggest that the success rate of exclusive breastfeeding in the study group was higher than that in the control group (94\% vs $72 \%$ ), the incidence of postpartum adverse events was lower than that of the control group ( $6 \%$ vs $26 \%$ ), and the SDS score and SAS score after nursing were lower than those in the control group $(\mathrm{P}<0.05)$. The findings reflect the positive influence of extended breastfeeding care on the maternal physiological and psychological state. The results are consistent with other studies $[11,12]$.

\section{Conclusion}

In conclusion, we demonstrated that extended breastfeeding care can improve the success rate of exclusive breastfeeding, improve maternal mood and reduce the incidence of postpartum 
adverse events. This type of extended care has a positive impact on maternal psychology and physiology, so it should be actively applied in clinical practice.

\section{References}

1. Tingting Huang (2020) Effect of extended pure breastfeeding nursing intervention on maternal physiological and psychological state. Electronic Journal of Clinical Medical Literature 7(22): 122.

2. Ugboaja J, Berthrand N, Igwegbe A, Amaka L Obi-Nwosu (2013) Barriers to postnatal care and exclusive breastfeeding among urbanwomen in southeastern Nigeria. Nigerian Medical Journal 54(1): 45-50.

3. Ambikapathi R, Kosek M N, Lee G O, Cloupas Mahopo, Crystal L Patil, et al. (2016) How multiple episodes of exclusive breastfeeding impact estimates of exclusive breastfeeding duration: report from the eight-site MAL-ED birth cohort study. Maternal and Child Nutrition 12(4): 740756.

4. Weiqun Shi, Shiyu Chen, ShaoxiaFeng (2018) Effect of extended pure breastfeeding nursing intervention combined with psychological nursing on parturient. Journal of Qilu Nursing 24(10): 44-46.

5. Rosane Siqueira Vasconcellos Pereira, Maria Inês Couto de Oliveira, Carla Lourenço Tavares de Andrade, Alexandre dos Santos Brito (2010) Factors associated with exclusive breastfeeding: The role of primary health care. Cad Saude Publica 26(12): 2343-2354.

6. Alina T I T, Manan W M W A, Isa B M (2016) The extended Theory of Planned Behavior in explaining exclusive breastfeeding intention and behavior among women in Kelantan, Malaysia. Nutr Res Pract 10(1): 49-55.
7. Ekubay M, Berhe A, Yisma E (2018) Initiation of breastfeeding within one hour of birth among mothers with infants younger than or equal to 6 months of age attending public health institutions in Addis Ababa, Ethiopia. International Breastfeeding Journal 13(1): 4.

8. Susan Kinney Davis, Jaynelle F Stichler, Debra M Poeltler (2010) Increasing Exclusive Breastfeeding Rates in the Well-Baby Population: An Evidence-Based Change Project. Nurs Womens Health 16(6): 460470.

9. Alves AL, Oliveira MI, Moraes JR (2013) Breastfeeding-Friendly Primary Care Unit Initiative and the relationship with exclusive breastfeeding. Revista De Saúde Pública 47(6): 1130-1140.

10. Zahra S, Monireh A, Easa M, Parsay Susan (2015) Successful breastfeeding mothers' experiences of the difficulties of exclusive breastfeeding. Acta Medica Mediterranea 31(7): 1479-1487.

11. Crume T L, Ogden L, Maligie M, Shelly Sheffield, Kimberly J Bischoff, et al. (2011) Long-Term Impact of Neonatal Breastfeeding on Childhood Adiposity and Fat Distribution Among Children Exposed to Diabetes In Utero. Diabetes Care 34(3): 641-645.

12. Santana Géssica S, Giugliani E R J, Vieira T d O, Graciete O Vieira (2018) Factors associated with breastfeeding maintenance for 12 months or more: a systematic review. J Pediatr (Rio J) 94(2): 104-122. 